\title{
Successful application of next-generation sequencing for pre-natal diagnosis in a pedigree with chronic granulomatosis disease
}

\author{
FANG PENG, LILI ZHONG, BING ZHANG, RUNYING ZOU, SHENGDAN NIE, \\ XIN TIAN, SHAOYANG DENG and XIANGLING HE
}

Department of Hematology and Oncology, Children's Medical Center, Hunan Provincial People's

Hospital/The First Affiliated Hospital of Hunan Normal University, Changsha, Hunan 410005, P.R. China

Received May 16, 2018; Accepted November 7, 2018

DOI: $10.3892 /$ etm.2019.7318

\begin{abstract}
The present study describes the successful application of next-generation sequencing (NGS) in the clinical diagnosis, pathogenic gene identification, treatment and pre-natal diagnosis in a pedigree with chronic granulomatosis disease (CGD). A 36-day-old infant, born to non-consanguineous Chinese parents, was admitted to hospital due to a neck lump for 10 days. A blood sample was collected for NGS to identify the molecular etiology. Sanger sequencing was performed for the patient and his relatives, including the parents. Amniotic fluid exfoliative cells from the mother were collected for pre-natal diagnosis at week 16 of a subsequent pregnancy. A novel c.1520_1521del, p.Lys508Aspfs*10 (NM_000397) variant in the cytochrome b-245 $\beta$ chain (CYBB) gene was identified in the proband, while the mother and the proband's 1-year-old sister were heterozygotes at this site. Karyotype analysis indicated that the fetus of the subsequent pregnancy was male. Sanger sequencing of amniotic cell DNA revealed that the fetus did not have the CYBB abnormality at the site. The results of the present study suggest that the variant in the CYBB gene was the cause of CGD in this pedigree and that pre-natal diagnosis using NGS is an effective method for providing genetic counseling to pedigrees with CGD.
\end{abstract}

\section{Introduction}

Chronic granulomatous disease (CGD) is a rare primary immunodeficiency disease caused by reduced nicotinamide adenine dinucleotide phosphate oxidase defects, which may impair the ability of phagocytes to kill peroxidase-positive bacteria and fungi, resulting in repeated and severe infections (1). The most

Correspondence to: Dr Xiangling He, Department of Hematology and Oncology, Children's Medical Center, Hunan Provincial People's Hospital/The First Affiliated Hospital of Hunan Normal University, 61 Jiefang West Rd, Changsha, Hunan 410005, P.R. China

E-mail: hexiangl@163.com

Key words: next-generation sequencing, cytochrome b-245 $\beta$ chain, chronic granulomatosis disease, pre-natal diagnosis common infection sites include the lungs, skin, lymph nodes and liver (1). For patients with CGD, an excessive inflammatory reaction gradually forms a granuloma; however, the clinical manifestations are varied, which makes early diagnosis difficult $(2,3)$. US and European statistics have reported incidence rates of CGD of 1/200,000-1/250,000 (4,5). At present, there is no precise data regarding the incidence of CGD in China; however, as the attention paid to primary immunodeficiency diseases increases and the assessment strategies improve, an increasing number of CGD diagnoses are made $(6,7)$. As the prognosis of this disease is poor, it is important to perform pedigree analyses and pre-natal diagnoses in affected families. In the present study, the clinical manifestations, pathogenic gene mutations and patterns of inheritance were assessed in a family with CGD to demonstrate the importance of next-generation sequencing (NGS) for the pre-natal diagnosis of this disease.

\section{Materials and methods}

Patient information. The proband, a 36-day-old male, was born to non-consanguineous Chinese parents. He was admitted to the hospital due to discovery of a neck lump 10 days previously. In the neo-natal period, the proband had been hospitalized at the neo-natal department of a local hospital due to neo-natal hyperbilirubinemia, neo-natal pneumonia and congenital hypothyroidism. He received oral levothyroxine tablets following discharge from the hospital. The mother was 'gravida 3, para 3', and the patient was the third child. The first child of the proband's parents, a male, died when he was 7 months old due to severe pneumonia, severe sepsis, septicemia (due to Burkholderia cepacia), intracranial infection and multiple organ failure. The second child of the proband's parents was a healthy 1-year-old female. The proband was born at week 39 of gestation with a birth weight of $3.7 \mathrm{~kg}$. No CGD-associated mutation within the family was known. Approximately 10 months after the birth of the proband, the mother became pregnant again.

Accessory examination. Routine laboratory examinations on blood, urine and stool samples were conducted. Blood tests were conducted using the Mindray blood cell analyzer (model no. BC-5000; Shenzhen Mindray Bio-Medical Electronics Co., Ltd., Shenzhen, China). Urinalysis was tested 
by manual standard methods (8) combined with dry chemistry test paper. Following the addition of isotonic saline to slides containing stool samples, they were view in several random fields for direct smear light microscopy using a magnification of $\mathrm{x} 10$ or $\mathrm{x} 40$ as appropriate. Erythrocyte sedimentation rate [ESR; the Westergren method (9)], C-reactive protein (CRP) and $\mathrm{T}$ cells with interferon- $\gamma$ release assays were performed. CRP content was measured with the Full C-Reactive Protein Quantification Test kit (Shanghai Upper Bio-Tech Pharma Co., Ltd., Shanghai, China) according to the manufacturer's protocol. $T$ cells with interferon- $\gamma$ release assays were conducted using the TB-IGRA kit (cat. no. TB-0296; WANTAI BioPharm Co., Ltd., Beijing, China). A chest radiograph was also performed. Immune globulin detection was measured by immuno-nephelometry using the BNII instrument (Dade Bering Inc., Deerfield, IL, USA), and a biopsy of the cervical lymph nodes was conducted. A biopsy of the cervical lymph nodes and immune globulin detection were conducted.

Measurement of the respiratory burst activity. In brief, $1 \mathrm{ml}$ peripheral blood was collected in a heparin anti-coagulant tube and was sent for detection within $2 \mathrm{~h}$. Phorbol ester (cat. no. P8139) and dihydrorhodamine (DHR; cat. no. D1054) were purchased from Sigma-Aldrich (Merck KGaA, Darmstadt, Germany). Ammonium chloride, EDTA and potassium carbonate were provided by Gold Wall Reagent Co. Ltd. (Kunshan, China). A working solution of hemolysin was composed of $8.29 \mathrm{~g}$ ammonium chloride, $0.037 \mathrm{~g}$ EDTA, $1.0 \mathrm{~g}$ potassium carbonate and 11 deionized water. As for flow cytometry, the analysis was performed using CELLQuest software (version 5.1; BD Biosciences, San Jose, CA, USA). The detection was performed as described by Richardson et al (10). Briefly, three samples were taken were placed in separate polypropylene tubes and were used as stimulated, resting and reagent blank tests. The tubes were successively incubated with working DHR solution $(30 \mu \mathrm{g} / \mathrm{ml})$ at $37^{\circ} \mathrm{C}$ for $5 \mathrm{~min}$ and FACS lysing solution (BD Biosciences) at room temperature for $10 \mathrm{~min}$. Following the centrifugation of the samples at $150 \mathrm{x} g$ at room temperature for $5 \mathrm{~min}$, they were washed with PBS and then fixed with $1 \%$ paraformaldehyde at $4^{\circ} \mathrm{C}$ for $30 \mathrm{~min}$ to stabilize the cells. To measurement the activity, the instrument was set up with a reagent blank sample and neutrophils were selected by gating. The negative contrast was adjusted to $10^{1}$ within the histogram using FL1 as the abscissa. Data were collected from all tubes, with 3,000 granulocytes obtained for each sample. During analysis, granulocytes were selected and displayed in a specific area using the forward angle and the lateral angle. A DHR fluorescence histogram was obtained in the gate and the average number of fluorescent signals was recorded.

Genetic sequencing. To determine the molecular cause of the disease in the proband, genetic sequencing, including NGS and Sanger sequencing, was performed. NGS was performed as previously described (11), with the modification that only 310 genes were captured. A gene library was prepared using peripheral blood, detected with the Agilent Bioanalyzer 2100 (Agilent Technologies, Inc., Santa Clara, CA, USA), and sequenced using the Illumina Hiseq2500 platform (Illumina, Inc., San Diego, CA, USA). The sequencing data were then processed using BclToFastq software (version 2;
Illumina, Inc.) and aligned with the National Center for Biotechnology Information human reference genome (HG-19) using the Burrows-Wheeler aligner $(12,13)$. Genome Analysis Toolkit software (GATK-3.7 software; Broad Institute, Cambridge, MA, USA) was used for identifying single nucleotide polymorphisms and insertion-deletions in the sequences. Protein biological function was predicted by using MutationAssessor (14), Provean (15) and PolyPhen-2 (16).

For Sanger sequencing, genomic DNA was extracted with Qiagen FlexiGene DNA kit (cat. no. 512206; Qiagen GmbH, Hilden, Germany) according the protocol provided by the manufacturer. The DNA was used for amplification was performed according with the method described previously (11) using an annealing temperature of $58^{\circ} \mathrm{C}$. The primers used were $5^{\prime}$-AGG GTGCCTTGGTTAGAATAGC-3' (forward) and 5'-TCTGTT GGGCATGAATTCAATC-3' (reverse) to generate a product $362 \mathrm{bp}$ in length. The polymerase chain reaction (PCR) products were sequenced using an ABI 3730XL (Thermo Fisher Scientific, Inc., Waltham, MA, USA) and analyzed with DNASTAR 5.0 software (DNASTAR, Inc., Madison, WI, USA).

Pre-natal diagnosis of the fetus. For pre-natal diagnosis, $30 \mathrm{ml}$ amniotic fluid was extracted at week 18 of pregnancy and was sent to the laboratory in a sterile culture tube. Following centrifugation, amniotic fluid DNA was extracted using the Qiagen FlexiGene DNA kit and PCR amplification was performed as mentioned above. In addition, the chromosomes were analyzed to identify the sex of the fetus. Briefly, $25 \mathrm{ml}$ amniotic fluid was centrifuged for $10 \mathrm{~min}$ at $295 \mathrm{x}$ g and room temperature. After the supernatant was discarded, amniotic fluid culture medium (CHANG Amnio $^{\circledR}$; Irvine Scientific Sales Company, Inc., Santa Ana, CA, USA) was added to prepare cell suspension. The cell suspension was inoculated in culture flask and cultured in $37^{\circ} \mathrm{C}$, $5 \% \mathrm{CO}_{2}$ incubator. The medium was changed for 7-8 days and the cells were harvested after 10 days. The cells were stained with Geimsa (Beijing Solarbio Science \& Technology Co., Ltd., Beijing, China) at room temperature for 3-5 min and karyotype analysis was performed under a phase contrast microscope at a magnification of $x 100$. These slides were examined systematically using an image analyzer system and Cyto-Vision software (version 3.93; Applied Imaging Corp., San Jose, CA, USA). DNA amplification products were sequenced using the ABI 3730XL (Thermo Fisher Scientific, Inc.) and analyzed using DNASTAR 5.0 software (DNASTAR, Inc.). In order to avoid experimental errors, PCR and sequencing experiments were repeated two times for the six samples.

\section{Results}

Clinical characteristics. To gain insight into the general condition of the proband, a physical examination was performed on admission. The infant had a normal development and was well nourished. The skin around his ears was red and skin damage was noted. Swollen lymph nodes (the size of peas to peanuts) were present at the back of the ears and the neck. The nodes were active and smooth, with no fusion, no pain reaction and no adhesion to the surrounding tissue.

Accessory examination results. In order to provide an accurate diagnosis, an additional examination was undertaken. 
A

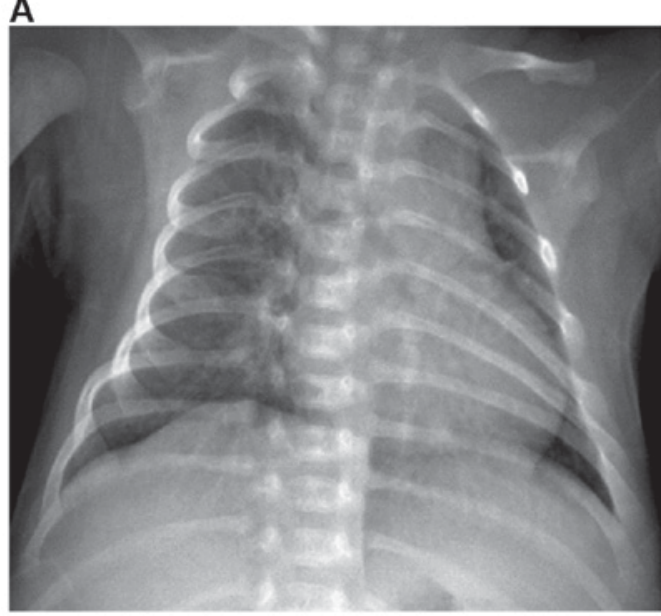

B

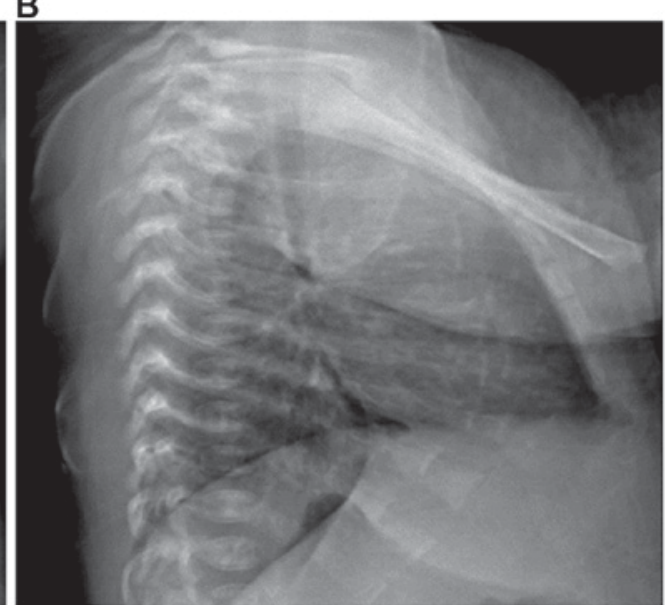

Figure 1. (A) Frontal and (B) lateral chest X-ray demonstrated thickened and blurred marking and scattered spots in the bilateral lung tissues, indicating bronchopneumonia.
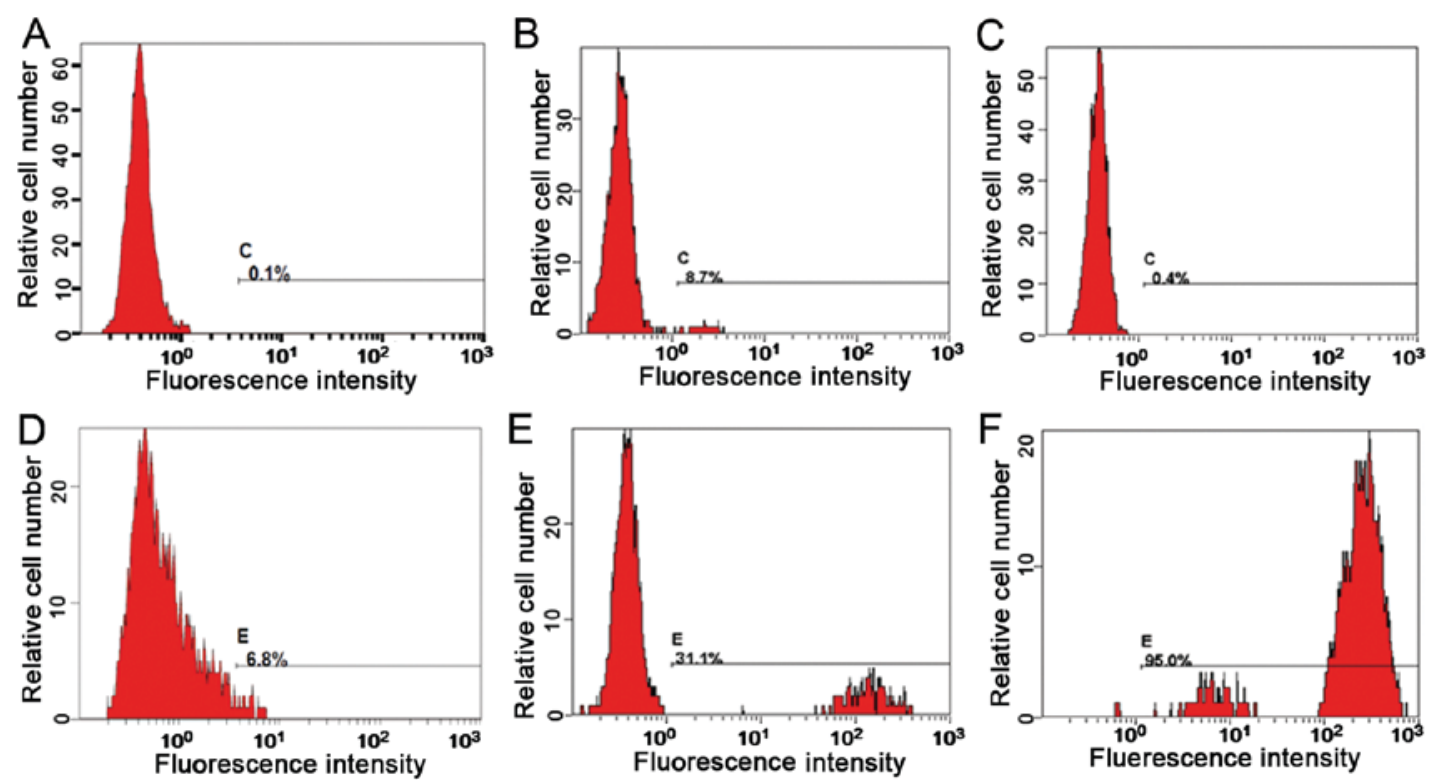

Figure 2. Comparison of neutrophil activation function prior to and after stimulation. (A) Neutrophil activation rate (inactivated) of the proband prior to stimulation. (B) Neutrophil activation rate of neutrophils after stimulation: No respiratory burst, positive expression. (C) Neutrophil activation rate of the mother prior to stimulation (inactivated). (D) the activation rate of neutrophils of the mother following stimulation revealed a bimodal reaction, indicating that the mother was a carrier. (E) Neutrophil activation rate of the father prior to stimulation (inactivated). (F) Neutrophil activation rate of the father after stimulation, indicating normal activation.

Routine blood results were as follows: White blood cells, $21.48 \times 10^{9} / 1$ (normal range, $\left.4-10 \times 10^{9} / 1\right)$; neutrophil ratio (N\%), $44.7 \%$ (normal range, 50-70\%); hemoglobin, 92 g/l (normal range, $110-140 \mathrm{~g} / \mathrm{l}$ ); and platelets, $416 \times 10^{9} / 1$ (normal range, $\left.100-300 \times 10^{9} / 1\right)$, indicating infection and mild anemia. Stool and urine were normal on routine examination. Chest radiography demonstrated thickened and blurred markings, as well as scattered spots in the bilateral lung tissues (Fig. 1), indicating bronchopneumonia. C-reactive protein was $43.4 \mathrm{mg} / 1$ (normal range, $<10 \mathrm{mg} / \mathrm{l}$ ) and the erythrocyte sedimentation rate was $67 \mathrm{~mm} / \mathrm{h}$ (normal range, $0-20 \mathrm{~mm} / \mathrm{h}$ ), suggesting the presence of bacterial infection. The detection of Mycobacterium tuberculosis in T cells with interferon- $\gamma$ release assay demonstrated that the level of Mycobacterium tuberculosis was $238.5 \mathrm{pg} / \mathrm{ml}$ (normal range, 0-14 pg/ml). Biopsy of a left cervical lymph node revealed lymph node granulomatous inflammation, with central necrosis, flakey debris and rapid coagulation necrosis, indicating tuberculosis. The biopsy staining was periodic acid-Schiff-negative and acid-fast stain-negative. Complete immunity detection revealed immunoglobulin ( $\mathrm{Ig}$ )A, $0.52 \mathrm{~g} / \mathrm{l}$ (normal range, 0.03-0.82 g/l), IgM, $1.10 \mathrm{~g} / 1$ (normal range, 0.15-1.09 g/l) and $\mathrm{IgG}, 6.74 \mathrm{~g} / \mathrm{l}$ (normal range, 7-14.8 g/l), which was considered as normal. No other abnormalities were noted.

Respiratory burst activity. Neutrophil samples were analyzed to determine any defects in the phagocytosis and oxidation function of neutrophils. Representative flow cytometry fluorescence intensity histograms are provided in Fig. 2 and the quantitative results are presented in Table I. The neutrophil 
Table I. Measurement of neutrophil function in the family (mean fluorescence intensity).

\begin{tabular}{lcccr}
\hline Subject & Control & $\begin{array}{c}\text { Phorbol 12-myristate } \\
\text { 13-acetate }\end{array}$ & $\begin{array}{c}\text { Neutrophil } \\
\text { activation rate (\%) }\end{array}$ & $\begin{array}{c}\text { Stimulation } \\
\text { index }\end{array}$ \\
\hline Proband & 0.452 & 1.2 & 6.8 & 0.4 \\
Proband's mother & 0.51 & 43.4 & 31.1 & 85.1 \\
Proband's father & 0.412 & 287 & 95.0 & 696.1 \\
\hline
\end{tabular}

activation rate and the stimulation index of the proband, the proband's father and the proband's mother were $6.8 \%$ and 0.4 , $95 \%$ and 696.1 , and $31.1 \%$ and 85.1 , respectively. It is assumed that the proband exhibited a neutrophil oxygen eruption deficiency.

Genetic analysis. In order to investigate the molecular etiology, genetic analysis, including NGS and Sanger sequencing, was performed. For the panel of 310 genes included, a total of 361 variants were identified. Among these variants, 262 were benign, 35 were likely benign, 59 were of uncertain significance and four were likely pathogenetic. The four variants were c.1447_1448del (NM_030917) in FIP1L1, p.Arg487Glyfs*3 (NM_000431) in MVK, c.863C $>$ T, p.Pro288Leu (NM_001002235) in SERPINA1, as well as c.880G>A, p.Asp294Asn and c.1520_1521del, p.Lys508Aspfs*10 (NM_000397) in cytochrome b-245 $\beta$ chain (CYBB), with a depth of $90,118,102$ and $133 x$, respectively. The variants in the first three genes are known to cause diseases different from the clinical features of the proband, therefore, they were excluded as they were unlikely to be pathogenic genes. CYBB is known to induce a disease similar to that of the patient $(17,18)$. Following filtering, the c.1520_1521del, p.Lys508Aspfs"10 (NM_000397) variant of the CYBB gene in the proband was considered as the etiological factor. The proband's mother was a carrier and the father was wild-type. CYBB is located on the $\mathrm{X}$ sex chromosome and CYBB mutations have been previously reported to be the cause of the X-linked recessive CGD (19). A pedigree diagram of the family was presented in Fig. 3A. Sanger sequencing further validated the results of NGS (Fig. 3B-E). In addition, Sanger sequencing was applied to the elder sister and the aunt (the mother's sister) of the proband, which revealed that the sister was a carrier and the aunt possessed a wild-type genotype (Fig. 3F and G). In summary, the proband and his sister possessed a c.1520_1521del, p.Lys508Aspfs*10 (NM_000397) variant in the $\mathrm{CYBB}$ gene passed on from their mother.

Clinical outcome. Fever was noted on the day of admission, and thus, cephathiamidin, meropenem and vancomycin were administered to treat potential infection. Ig was used for symptomatic treatment. However, the fever did not subside, the swelling of the cervical lymph nodes was not reduced and the patient experienced seizures. The parents refused further treatment and discharged the child.

Pre-natal diagnosis. Molecular genetic diagnosis was performed for the proband; however, follow-up revealed that he had died 21 days after discharge. To prevent the mother from giving birth to another infant with the same disease, amniotic cells were collected and cultured for further analysis during a subsequent pregnancy. As demonstrated in Fig. 4, karyotype analysis indicated that the fetus was male. Sanger sequencing of amniotic cell DNA indicated no variant in the CYBB gene (Fig. 3G). Considering the pre-natal diagnosis result, the mother continued the pregnancy and gave birth to a healthy infant.

\section{Discussion}

CGD has two genetic types: Autosomal recessive CGD and $\mathrm{X}$-linked recessive CGD. X-linked recessive CGD accounts for 65-70\% of all CGD cases (5). In the present study, the proband had a healthy elder sister and an elder brother that exhibited repeated infection at the age of 7 months and died due to severe pneumonia and Klebsiella sepsis. Considering the clinical information, it is probable the elder brother also had CGD. The clinical manifestations of this disease are variable; infection of the respiratory tract, skin, lymph nodes and digestive tract, and granuloma are the most common features (5). The proband of the present study initially presented with neck lymphadenopathy but without any other obvious symptoms. He had been vaccinated with Bacillus Calmette-Guérin against tuberculosis, but had no scar. On the basis of T-cell detection and lymph node biopsy results, he was highly suspected to have a tuberculosis infection. The family history suggested that a primary immunodeficiency disease was involved. Within this pedigree, children that present with lymph node enlargement, pathogen infection and potential immunodeficiency, CGD should be considered as a possible cause in addition to blood system disorders and connective tissue diseases.

Laboratory analysis demonstrated that the number of leukocytes was increased in the proband, suggesting the presence of infection. Ig was also was slightly increased. An increase of $\gamma$-globulin is a common phenomenon in children and it has implications for further clinical symptoms (20). The diagnosis of CGD is based on direct determination of the production of superoxide. Dihydrorhodamine flow cytometry has gradually replaced nitroblue tetrazolium analysis due to its relatively simple operation, and its ability to distinguish the $\mathrm{X}$ linkage and autosomal patterns by flow cytometry. In addition, to a certain extent, the prognosis of pediatric patients with CGD may be estimated using this method. It was previously demonstrated that the activity of residual neutrophils is positively correlated with survival rate (21). The activity of neutrophils in this case was significantly decreased, the onset was early, and the prognosis was poor, which is consistent with previous report (21). Flow cytometry is sensitive to even 
A
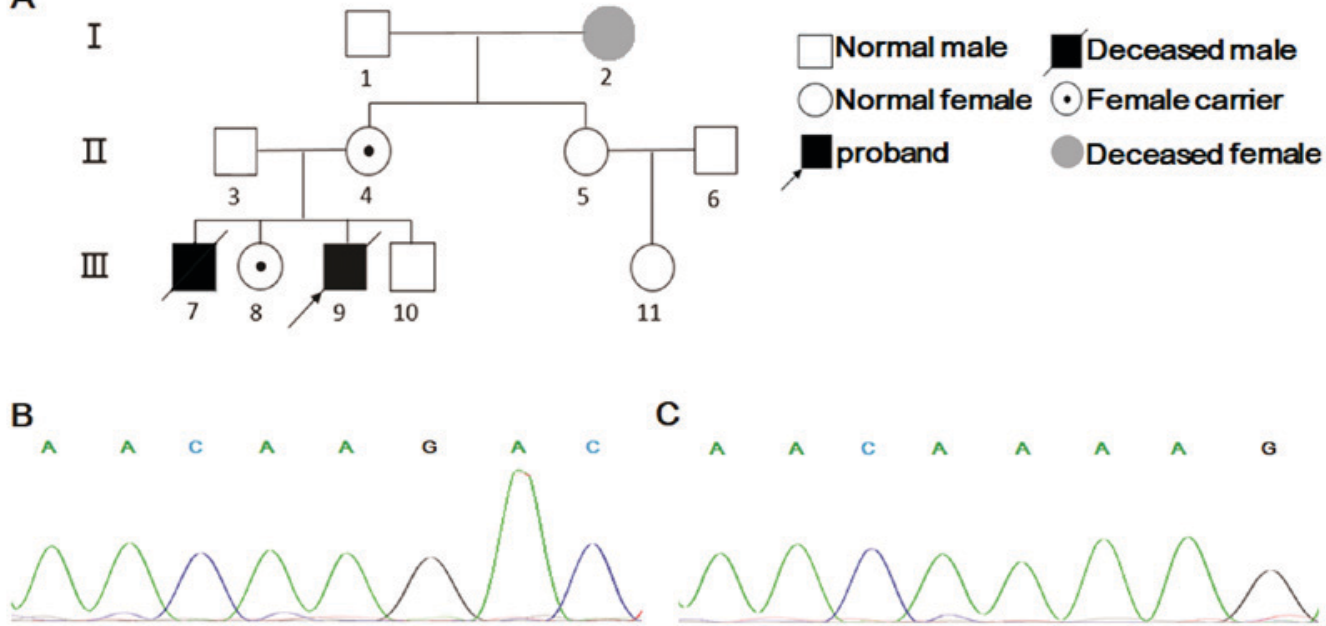

C
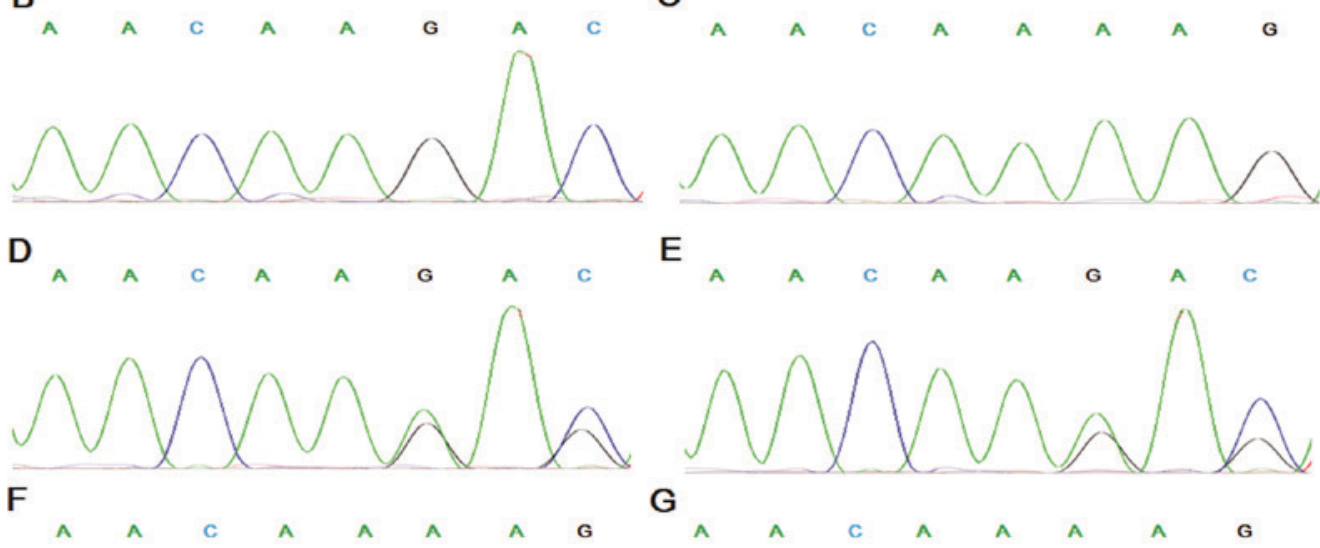

G

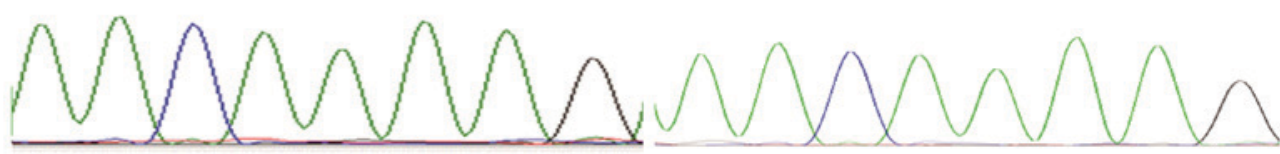

Figure 3. Pedigree diagram and Sanger sequencing results. (A) Pedigree of the family. (B) The proband had a c.1520_1521del mutation in the CYBB gene, while the sequence of (C) the father resembled wild-type. (D) The mother and (E) sister of the proband had a heterozygote c.1520_1521del mutation in the CYBB gene, whereas (F) the sequence of the proband's aunt resembled a reference sequence. (G) The fetus of the mother's new pregnancy had wild type CYBB gene.

a small change in the number of functional neutrophils (21). In the present study, the proband was diagnosed using this method, and his mother and sister were screened and identified as carriers. Pre-natal diagnosis for primary immunodeficiency disease by gene detection has been applied for numerous years (22).

CGD may be caused by mutations in the CYBB gene, which is $30 \mathrm{~kb}$ in length and contains 13 exons. The mutations are widespread, without hot-spot mutation. No associations between genotype and phenotype have been identified thus far. Genetic analysis confirmed that the proband of the present study possessed the CYBB gene mutation c.1520_1521del, (NM_000397). This is a novel variant and may cause a frameshift from Lys at the 508 amino acid site. This variant was identified as the molecular cause of the disease and abnormal neutrophil respiratory burst function was also observed. In recent years, flow cytometry has been used for the pre-natal diagnosis of CGD (23). Flow cytometry is a fast and sensitive method for detecting CGD; however, the accuracy of this method requires further evaluation (24). In the present case, the mother desired to have more children; therefore, genetic testing was performed using amniotic fluid cell culture during her subsequent pregnancy. Chromosome karyotype analysis and genetic testing suggested that the mother was a carrier and the fetus was a normal male. Peripheral blood was collected

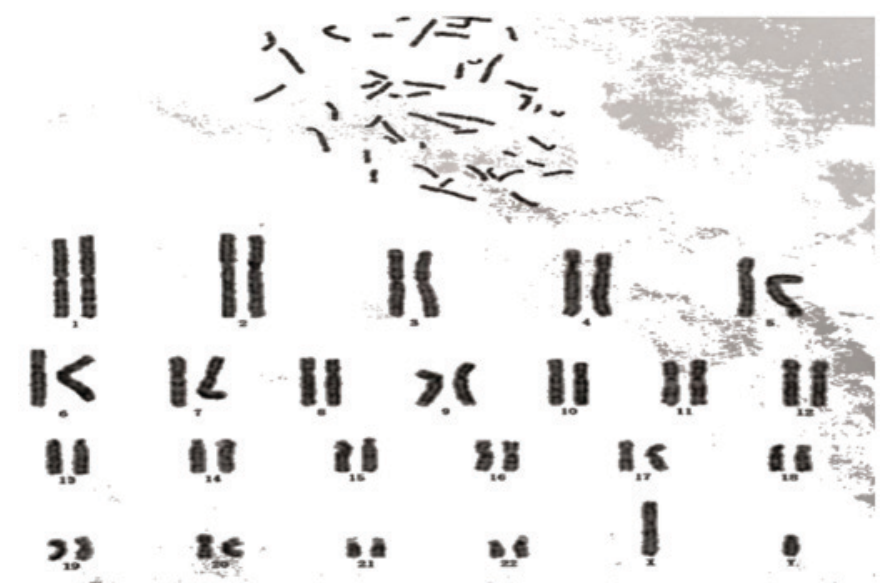

Figure 4. Chromosome karyotype analysis revealed that fetus of the proband's mother's new pregnancy was male, with a 46,XY karyotype.

from the infant following birth and the genetic test results were also normal. Testing was also performed on other members of the pedigree. The sister of the proband was also a mutation carrier, while the proband's aunt had a normal genotype.

A method of CGD treatment that may cure the disease is immune reconstruction, including hematopoietic stem cell 
transplantation (HSCT) and gene therapy. Although HSCT has various difficulties, including the retrieval of a suitable match and graft-versus-host disease following transplantation, HSCT has been used to eradicate CGD in numerous cases $(25,26)$. Gene therapy is still in the experimental stages, and it may take a number of years until it is commonly used as a treatment for CGD worldwide (27).

\section{Acknowledgements}

Not applicable.

\section{Funding}

No funding received.

\section{Availability of data and materials}

The data for present study are available from the corresponding author on reasonable request.

\section{Authors' contributions}

LZ and XH designed the study. FP collected the clinical data, performed the experiments, and drafted and wrote the manuscript. RZ participated in clinical data collection. All authors read and approved the final version of the manuscript.

\section{Ethics approval and consent to participate}

The present study was approved by the ethics committee of Hunan Provincial People's Hospital (Changsha, China) and informed consent was obtained from the aunt and the parents of the proband.

\section{Patient consent for publication}

Not applicable.

\section{Competing interests}

All authors declare that they have no competing interests.

\section{References}

1. Segal BH, Leto TL, Gallin JI, Malech HL and Holland SM: Genetic, biochemical, and clinical features of chronic granulomatous disease. Medicine 79: 170-200, 2000.

2. Roos D and de Boer M: Molecular diagnosis of chronic granulomatous disease. Clin Exp Immunol 175: 139-149, 2014.

3. Rawat A, Vignesh P, Sharma A, Shandilya JK, Sharma M, Suri D, Gupta A, Gautam V, Ray P, Rudramurthy SM, et al: Infection profile in chronic granulomatous disease: A 23 -year experience from a tertiary care center in north India. J Clin Immunol 37: 319-328, 2017

4. Winkelstein JA, Marino MC, Johnston RB Jr, Boyle J, Curnutte J, Gallin JI, Malech HL, Holland SM, Ochs H, Quie P, et al: Chronic granulomatous disease. Report on a national registry of 368 patients. Medicine (Baltimore) 79: 155-169, 2000.

5. van den Berg JM, van Koppen E, Ahlin A, Belohradsky BH, Bernatowska E, Corbeel L, Español T, Fischer A, Kurenko-Deptuch M, Mouy R, et al: Chronic granulomatous disease: The European experience. PLoS One 4: e5234, 2009.

6. Arnold DE and Heimall JR: A review of chronic granulomatous disease. Adv Ther 34: 2543-2557, 2017.
7. Beghin A, Comini M, Soresina A, Imberti L, Zucchi M, Plebani A, Montanelli A, Porta F and Lanfranchi A: Chronic granulomatous disease in children: A single center experience. Clin Immunol 188: 12-19, 2018

8. Grinstead GF, Scott RE, Stevens BS, Ward VL and Wilson DM: The Ames Clinitek 200/Multistix 9 urinalysis method compared with manual and microscopic methods. Clin Chem 33: 1660-1662, 1987.

9. Shelat SG, Chacosky D and Shibutani S: Differences in erythrocyte sedimentation rates using the Westergren method and a centrifugation method. Am J Clin Pathol 130: 127-130, 2008.

10. Richardson MP, Ayliffe MJ, Helbert M and Davies EG: A simple flow cytometry assay using dihydrorhodamine for the measurement of the neutrophil respiratory burst in whole blood: Comparison with the quantitative nitrobluetetrazolium test. J Immunol Methods 219: 187-193, 1998.

11. Jin D, Yu T, Zhang L, Wang T, Hu J, Wang Y and Yang XA: Novel NFU1 variants induced MMDS behaved as special leukodystrophy in Chinese sufferers. J Mol Neurosci 62: 255-261, 2017.

12. Li H and Durbin R: Fast and accurate long-read alignment with Burrows-Wheeler transform. Bioinformatics 26: 589-595, 2010.

13. $\mathrm{Li} \mathrm{H}$ and Durbin R: Fast and accurate short read alignment with Burrows-Wheeler transform. Bioinformatics 25: 1754-1760, 2009.

14. Reva B, Antipin Y and Sander C: Predicting the functional impact of protein mutations: Application to cancer genomics. Nucleic Acids Res 39: e118, 2011.

15. Choi Y and Chan AP: PROVEAN web server: A tool to predict the functional effect of amino acid substitutions and indels. Bioinformatics 31: 2745-2747, 2015.

16. Adzhubei IA, Schmidt S, Peshkin L, Ramensky VE, Gerasimova A, Bork P, Kondrashov AS and Sunyaev SR: A method and server for predicting damaging missense mutations. Nat Methods 7: 248-249, 2010.

17. Iwasaki J, Kondo T, Darmanin S, Ibata M, Onozawa M, Hashimoto D, Sakamoto N and Teshima T: FIP1L1 presence in FIP1L1-RARA or FIP1L1-PDGFRA differentially contributes to the pathogenesis of distinct types of leukemia. Ann Hematol 93: 1473-1481, 2014.

18. Silva D, Oliveira MJ, Guimaraes M, Lima R, Gomes S and Seixas S: Alpha-1-antitrypsin (SERPINA1) mutation spectrum: Three novel variants and haplotype characterization of rare deficiency alleles identified in Portugal. Respir Med 116: 8-18, 2016.

19. Roos D: Chronic granulomatous disease. Br Med Bull 118: 50-63, 2016.

20. Wolach B, Gavrieli R, de Boer M, van Leeuwen K, Berger-Achituv S, Stauber T, Ben Ari J, Rottem M, Schlesinger Y, Grisaru-Soen G, et al: Chronic granulomatous disease: Clinical, functional, molecular, and genetic studies. The Israeli experience with 84 patients. Am J Hematol 92: 28-36, 2017.

21. Kuhns DB, Alvord WG, Heller T, Feld JJ, Pike KM, Marciano BE Uzel G, DeRavin SS, Priel DA, Soule BP, et al: Residual NADPH oxidase and survival in chronic granulomatous disease. N Engl J Med 363: 2600-2610, 2010

22. Newburger PE, Cohen HJ, Rothchild SB, Hobbins JC, Malawista SE and Mahoney MJ: Prenatal diagnosis of chronic granulomatous disease. N Engl J Med 300: 178-181, 1979.

23. Kulkarni M, Gupta M and Madkaikar M: Phenotypic prenatal diagnosis of chronic granulomatous disease: A useful tool in the absence of molecular diagnosis. Scand J Immunol 86: 486-490, 2017.

24. Newburger PE: Comment on: Phenotypic prenatal diagnosis of chronic granulomatous disease: A useful tool in the absence of molecular diagnosis. Scand J Immunol 87: 57, 2018.

25. Ahlin A and Fasth A: Chronic granulomatous disease-conventional treatment vs. hematopoietic stem cell transplantation: An update. Curr Opin Hematol 22: 41-45, 2015.

26. Norman M, David C, Wainstein B, Ziegler JB, Cohn R, Mitchell R, O'Brien T, Russell S, Trahair T, Trickett A, et al: Haematopoietic stem cell transplantation for primary immunodeficiency syndromes: A 5-year single-centre experience. J Paediatr Child Health 53: 988-994, 2017.

27. De Ravin SS, Li L, Wu X, Choi U, Allen C, Koontz S, Lee J, Theobald-Whiting N, Chu J, Garofalo M, et al: CRISPR-Cas9 gene repair of hematopoietic stem cells from patients with X-linked chronic granulomatous disease. Sci Transl Med 9: pii: eaah3480, 2017.

This work is licensed under a Creative Commons Attribution-NonCommercial-NoDerivatives 4.0 International (CC BY-NC-ND 4.0) License. 